\title{
BRASEENDER
}

Trascender, Contabilidad y Gestión Núm. 9 (septiembre - diciembre del 2018). ISSN: 2448-6388. Universidad de Sonora. Departamento de Contabilidad. Reserva de Derechos 04-2015-04172070800-203.

\section{Análisis de los tipos de acoso y discriminación recogidos en los códigos de conducta de las empresas cotizadas en la Bolsa española en el mercado continuo a 31-12-2015}

\author{
Analysis of the types of harassment and discrimination included in the codes of conduct of companies listed \\ on the Spanish Stock Exchange on 12/31/2015
}

María Concepción Verona Martel ${ }^{1}$; Sara De León Santana ${ }^{2}$

\section{Resumen}

En este trabajo se analizan los diferentes tipos de acoso y discriminación recogidos en los códigos éticos de las empresas cotizadas en la Bolsa española a 31-12-2015. Los resultados muestran que las empresas incluyen una amplia variedad de tipos de acoso y discriminación en su código de conducta, entre los que destaca el rechazo a la discriminación por razón de sexo. Asimismo, el análisis por sectores pone de manifiesto que en todos los sectores no se da la misma importancia a los diferentes tipos de acoso y discriminación.

Palabras clave: códigos éticos, empresas cotizadas, tipos de acoso y discriminación en el trabajo.

DOI: https://doi.org/10.36791/tcg.v0i9.33

Recibido: 24 de junio de 2018.

Aceptado: 28 de agosto de 2018.

\section{Abstract}

In this paper we analyze the different types of harassment and discrimination included in the ethical codes of companies listed on the Spanish Stock Exchange on 12/31/2015. The results show that companies include a wide variety of types of harassment and discrimination in their code of conduct, among which the rejection of discrimination based on sex stands out. Likewise, the analysis by sectors shows that in all sectors, the different types of harassment and discrimination are not given the same importance.

Keywords: ethical codes, listed companies, types of harassment and discrimination at work.

\footnotetext{
${ }^{1}$ María Concepción Verona Martel es Doctora en Ciencias Económicas y Empresariales por la Universidad de Las Palmas de Gran Canaria (ULPGC), en la cual ocupa el puesto de Profesora Titular de Universidad. Correo: mariaconcepcion.verona@ulpgc.es

${ }^{2}$ Sara De León Santana es graduada en Turismo por la Universidad de Las Palmas de Gran Canaria (ULPGC). 


\section{Introducción}

La ética en los negocios es un tema preocupante y de gran actualidad. La crisis financiera en la que quedó inmersa la economía mundial en 2008 supone un estímulo adicional para la implementación de códigos éticos o códigos de conducta en las empresas, ya que en ellos se recogen principios y valores que ayudan en la toma de decisiones éticas en las organizaciones.

Los códigos de conducta, publicados en las páginas web oficiales de las empresas, se han convertido en una medida de señalización para todos los grupos de interés y el público en general del nivel de compromiso que tiene la empresa con la ética.

Entre los temas que recogen los códigos éticos de las empresas se encuentra la no tolerancia al acoso y discriminación en sus diferentes formas. Teniendo esto presente, el objetivo del este trabajo se centra en analizar el grado de presencia de los diferentes tipos de acoso y discriminación en los códigos éticos de las empresas cotizadas en la Bolsa española en el mercado continuo a fecha de último día de 2015, haciéndose el análisis tanto a nivel global como por sectores económicos.

Este trabajo se ha dividido en las siguientes secciones. Tras la introducción, en la sección segunda se hace una breve referencia a aspectos generales de los códigos de conducta y se indica el objetivo del estudio. En la sección tercera se recoge la muestra analizada y la metodología utilizada. La sección cuarta queda para los resultados obtenidos y la sección quinta recoge las conclusiones del trabajo.

\section{Códigos de conducta: aspectos generales. Objetivo del estudio}

Cortina (2003: 17) afirma que la ética es "un tipo de saber de los que pretende orientar la acción humana en el sentido racional; es decir, pretende que obremos racionalmente. A diferencia de los saberes preferentemente teóricos, contemplativos, a los que no importa en principio orientar la acción, la ética es esencialmente un saber para actuar de un modo racional. Pero no solo en un momento puntual [...] sino para actuar racionalmente en un conjunto de la vida”.

Este autor haciendo referencia a la empresa señala que la misma "no es simplemente una máquina o un agregado de átomos inconexos, sino que efectivamente tiene una estructura que la lleva a funcionar de forma organizada, entonces debe estar dotada de rasgos análogos a los que hemos reconocido como propios de un organismo humano. Esto significa que debe tomar conciencia de qué valores y metas deben orientar sus decisiones, porque son los que le ayudarán a ir conformando su identidad, un carácter propio de la organización. Y también que la organización, como tal, debe hacerse responsable de sus decisiones y de las consecuencias previsibles que de ellas se sigan" (Cortina, 1998: 128).

La empresa "puede y debe contribuir a la realización personal y a la mejora del entorno social” (Debeljuh, 2005: 50), por tanto, "todas sus acciones tienen una dimensión ética y son susceptibles de una valoración moral. Por ello, si la empresa no contribuye a desarrollar las virtudes humanas de todos aquellos que se relacionan con ella, deberá replantearse su propia existencia” (Debeljuh, 2005: 50).

La clave para que se consolide una cultura ética en la empresa está "en conseguir que los directivos y todos los agentes económicos asuman personalmente esas cualidades” (Regojo, 2014: 28).

Toda empresa que "de verdad se comprometa con la ética ha de transmitir ese mensaje a toda la organización y también a aquellos que se relacionan con ella" (Debeljuh, 2005: 51), y un vehículo eficaz para hacerlo es "mediante un código escrito que defina la misión de la compañía, sus valores y las reglas de comportamiento" (Debeljuh, 2005: 51). En esta misma línea se pronuncia Lozano Aguilar (2007: 227) al afirmar que "el desarrollo de la ética en las organizaciones es un camino en el que el primer paso es la elaboración de un código de ética”. 
Los códigos éticos, también llamados códigos de conducta, han demostrado ser "un buen instrumento para mejorar el compromiso ético de las personas en la organización y de la propia organización con la sociedad” (Lozano Aguilar, 2007: 235), aunque no se pueden considerar el elemento clave para solucionar todos los problemas de la organización pues "no constituyen un blindaje frente al fraude" (Iranzo, 2004: 268), ya que "el solo hecho de contar con un código de conducta no hace éticas a las personas ni, por ende, a las organizaciones” (Debeljuh, 2005: 51). No obstante, su elaboración e implantación en la empresa se puede considerar como un paso importante de la misma hacia el comportamiento ético en el desempeño de sus actividades. Por tanto, "un código solo no hace una organización ética. El código debe ser un elemento más de la integración e institucionalización de la ética en la organización” (Lozano Aguilar, 2004: 163).

En palabras de Valor y De la Cuesta González (2007: 20) un código ético intenta definir "la filosofía y los valores de la empresa, fijando reglas para enfrentarse a ciertos dilemas o asuntos delicados que pueden surgir en la dirección y gestión de la misma”.

El gran desafío que se le presenta a los códigos de ética es conseguir que "sus principios se traduzcan en realidades concretas. Para eso es importante, de una parte, que su contenido encierre verdaderos valores morales y, de otra, que su elaboración, implantación y seguimiento favorezcan el desarrollo de las virtudes morales” (Debeljuh, 2005: 52). El método con que se "elabora un código, así como la racionalidad que subyace en él, influye de forma decisiva en su efectividad; pero la efectividad del código para mejorar la conducta ética de la organización debe ir acompañada de otras actuaciones a lo largo y ancho de toda la organización” (Lozano Aguilar, 2004: 163).
En las empresas se “deben diseñar y realizar procesos que contribuyan al desarrollo efectivo del código ético. Las comisiones éticas, los departamentos de cumplimiento y los cursos de formación ética son buenos mecanismos para el desarrollo eficaz de los códigos éticos” (Lozano Aguilar, 2004: 221). Asimismo, que "la alta dirección impulse la integración de los códigos en la gestión diaria de la compañía es el mejor camino para la efectividad de los códigos a la hora de orientar la conducta de las personas que trabajan en la organización; y eso requiere que los valores propuestos en el código se concreten en políticas, normas y procedimientos en la organización misma” (p. 209). En esta misma línea se pronuncia Debeljuh (2005: 53), quién hace hincapié en el hecho de que para una correcta implementación del código ético en una empresa "es necesaria una buena política de comunicaciones que posibilite a cada individuo conocer con profundidad el sentido de los valores de la compañía para que después quiera, libremente, escogerlos en las acciones concretas”, al mismo tiempo que señala como requisito indispensable "el valor de la ejemplaridad y de la coherencia de la alta dirección para que, a partir del propio compromiso con los valores morales, puedan exigirlo a los demás” (Debeljuh, 2005: 54).

Por tanto, se puede afirmar que la utilidad de los códigos éticos de una empresa está estrechamente vinculada "al desarrollo de virtudes de las personas involucradas, a una adecuada política de comunicación y a un firme liderazgo, comprometido con los valores morales” (Debeljuh, 2005: 48).

El carácter público del código ético es un rasgo esencial en un auténtico código ético (Lozano Aguilar, 2007: 233). Este autor señala que si bien hay empresas que consideran que el código ético es un documento interno que no debe ser conocido por el público, a su 
juicio "esto es un error puesto que una de las funciones esenciales del código ético es la de generar legitimidad entre los Stakeholders externos de la organización, algo que obviamente se pierde si no se da a conocer” (Lozano Aguilar, 2007: 234).

En España los códigos éticos son documentos de elaboración totalmente voluntaria por parte de las empresas, y su divulgación a personas ajenas a las mismas también lo es, pues las empresas los pueden elaborar y darlos a conocer sólo a sus empleados. Sin embargo, con el paso de los años se ha podido observar que las empresas que no hacían visible para el público en general su código de conducta han cambiado de opinión y actualmente tienen publicado en su página web oficial el mismo en acceso libre.

La responsabilidad de elaborar e impulsar el código ético de la organización recae en el consejo de administración de la empresa, siendo dicho código de obligado cumplimiento para todos los empleados de la misma, entendiendo por empleados a los administradores, ejecutivos de cualquier nivel y personal en general de la empresa.

En la elaboración del código de conducta de la empresa se debe hacer partícipe a todos los estamentos de la organización, para que éste sea asumido con más facilidad por todos sus miembros. Además, los códigos éticos deben ser documentos dinámicos para lo cual se han de renovar con el paso de los años, para así adaptarlos a la realidad que rodea a la empresa en cada momento.

Verona-Martel y Sosa-Breyer (2014: 36) analizaron el contenido de los códigos éticos de las empresas cotizadas en la Bolsa española y concluyeron que el contenido de los mismos se puede dividir en tres bloques: a) ítems referidos a la relación del empleado con la empresa, b) ítems referidos a la relación de la empresa con el empleado, y c) ítems referidos a la relación de la empresa con el entorno.
El primer bloque es el que tiene un mayor peso en los códigos éticos mientras que el tercero es el que tiene una menor importancia; el ítem de rechazo al acoso y discriminación se encuentra en el primer bloque (p. 36).

Los beneficios que reporta un código ético para la empresa que lo posee "tal vez no consten en la cuenta de resultados, pero es innegable que están presentes en sus ganancias, y más aún, en el modo de conseguirlas. Un código de ética se ocupa no tanto de la manera de gestionar la empresa en aras de beneficios medidos o cuantificables, sino de cómo obtenerlos a través de la mejora de las personas” (Debeljuh, 2005: 49).

Regojo (2014: 103) señala que una de las características esenciales de cualquier institución, como es el caso de una empresa, "es estar constituida por personas”, lo que conlleva que una de sus finalidades esenciales sea "respetar a los que en ella trabajan y contribuir a su desarrollo humano y profesional". Por tanto, las prácticas de acoso y discriminación no se deben tolerar.

En España diferentes normativas hacen referencia al acoso y a la discriminación como prácticas que no se deben permitir. Así, el artículo 4 del Estatuto de los Trabajadores recoge los derechos laborales del trabajador. En dicho artículo, en el punto 2 apartados c), d) y e) se indica lo siguiente:

“2. En la relación de trabajo, los trabajadores tienen derecho: [...]

c) A no ser discriminados directa o indirectamente para el empleo, o una vez empleados, por razones de sexo, estado civil, edad dentro de los límites marcados por esta ley, origen racial o étnico, condición social, religión o convicciones, ideas políticas, orientación sexual, afiliación o no a un sindicato, así como por razón de lengua, dentro del Estado español. 
Tampoco podrán ser discriminados por razón de discapacidad, siempre que se hallasen en condiciones de aptitud para desempeñar el trabajo o empleo de que se trate.

d) A su integridad física y a una adecuada política de seguridad e higiene.

e) $\mathrm{Al}$ respeto de su intimidad y a la consideración debida a su dignidad, comprendida la protección frente al acoso por razón de origen racial o étnico, religión o convicciones, discapacidad, edad u orientación sexual, y frente al acoso sexual y al acoso por razón de sexo”.

Por su parte la Constitución Española recoge en el artículo 14 que: "Los españoles son iguales ante la ley, sin que pueda prevalecer discriminación alguna por razón de nacimiento, raza, sexo, religión, opinión o cualquier otra condición o circunstancia personal o social”. Asimismo, el artículo 15 señala el "derecho a la integridad física y moral”, el artículo 16 "garantiza la libertad ideológica”, y el artículo 28 señala que "todos tienen derecho a sindicarse libremente".

Si se acude al Código Penal español se puede observar que son considerados delitos tanto el acoso sexual (art. 184) como el acoso psicológico en el trabajo (art. 173.1).

En 2007 se aprueba en España la Ley Orgánica $3 / 2007$, de 22 de marzo, para la igualdad efectiva de mujeres y hombres recogiendo en su artículo 3 que: "El principio de igualdad de trato entre mujeres y hombres supone la ausencia de toda discriminación, directa o indirecta, por razón de sexo, y, especialmente, las derivadas de la maternidad, la asunción de obligaciones familiares y el estado civil”. El artículo 7.3 señala que "se considerarán en todo caso discriminatorios el acoso sexual y el acoso por razón de sexo”, y en el artículo 8 se señala que "constituye discriminación directa por razón de sexo todo trato desfavorable a las mujeres relacionado con el embarazo o la maternidad”.
Al ser el código ético el documento que recoge el comportamiento ético que se quiere que impere en la empresa es de esperar que los distintos tipos de acoso y discriminación señalados en las anteriores normativas sean recogidos también en el código de conducta de las empresas. Aunque el rechazo a las diferentes formas de acoso y discriminación estén recogidos en normativas el hecho ser incluidas en el código de conducta de la empresa supone un refuerzo adicional.

Teniendo presente todo lo anterior, y las palabras de Lozano Aguilar (2004: 18) "nos ocupamos de él [del código ético] por el absoluto convencimiento de su necesidad para el desarrollo no sólo de unas organizaciones más eficaces sino también de una sociedad más justa y próspera”, el objetivo del presente estudio queda definido en los siguientes términos:

Analizar los tipos de acoso y discriminación recogidos en los códigos de conducta de las empresas cotizadas en la Bolsa española en el mercado continuo a 31-12-2015.

\section{Muestra objeto de estudio y metodología utilizada}

3.1. Muestra objeto de estudio.

La muestra objeto de estudio en este trabajo está formada por los códigos éticos o códigos de conducta de las empresas cotizadas en la Bolsa española en el mercado continuo a 31 de diciembre de 2015. En esa fecha cotizaban 129 empresas pertenecientes a distintos sectores económicos.

Autores como García Sánchez et al. (2008: 106) consideran que una empresa tiene realmente un código de conducta cuando dicho documento se encuentra a disposición de cualquier interesado (accionistas, clientes, proveedores, etc.) en la página web corporativa, y este será el criterio que se va a seguir en este trabajo.

De las 129 empresas cotizadas en la Bolsa española en el mercado continuo a último día de diciembre de 2015, 
90 tienen código de conducta pero 5 de ellas no lo tienen visible para el público en general, por lo que la muestra a analizar en este estudio queda formada por 85 códigos éticos. Por tanto, a fecha de finales de 2015 el 65,89\% de las empresas cotizadas contaban con código ético visible. Se ha podido comprobar también que empresas que en años anteriores, por ejemplo en el año 2013 no tenían su código ético visible, a fecha de 2015 ya lo han hecho visible.

Verona-Martel y Sosa-Breyer (2014: 30) señalan en su estudio que a fecha del 2013 sólo el 48,78\% de las empresas cotizadas en la Bolsa española en el mercado continuo poseían código ético visible. Con lo cual se observa un incremento significativo del número de empresas que han elaborado y hecho público su código de conducta.

\subsection{Metodología utilizada.}

Para la realización del presente trabajo se han consultado los códigos éticos de las empresas cotizadas en la Bolsa española en el mercado continuo, los cuales se recogen en la página web oficial de la empresa.

En los códigos éticos se utilizan los términos acoso y discriminación en muchas ocasiones como sinónimos pero en este trabajo se ha optado por utilizar el término acoso para el acoso sexual, el acoso psicológico en el trabajo (mobbing) y el acoso físico y el término discriminación para los demás casos, es decir, discriminación por razón de sexo, por raza, por nacionalidad, por religión, por afiliación sindical, etc.

En el proceso de análisis de los códigos éticos se ha considerado que en dicho código se recogía el rechazo a un determinado tipo de acoso o discriminación cuando textualmente así se hacía constar.

\section{Resultados obtenidos}

4.1. Resultados obtenidos en relación a los tipos de acoso y discriminación cuyo rechazo se recoge en los códigos éticos de las empresas cotizadas en la Bolsa española en el mercado continuo a 31-12-2015.

A 31 de diciembre de 2015 sólo 85 empresas de las 129 que cotizaban en la Bolsa española en el mercado continuo poseían un código ético visible.

Las empresas cotizadas en Bolsa pertenecen a distintos sectores económicos y es de interés conocer si en todos los sectores de actividad las empresas dan la misma importancia a la posesión y publicación del código de conducta. En el cuadro 1 se recoge esta información utilizándose para ello la clasificación sectorial que utilizó la Bolsa de Madrid hasta el año 2018. 
Cuadro 1. Posesión o no de un código de conducta y sector de actividad al que pertenece la empresa

\begin{tabular}{|c|c|c|c|c|c|c|c|}
\hline $\begin{array}{l}\text { Sector de } \\
\text { actividad }\end{array}$ & $\begin{array}{c}\text { Petróleo } \\
\text { y } \\
\text { Energía }\end{array}$ & $\begin{array}{l}\text { Mat. Básicos, } \\
\text { Industria y } \\
\text { Construcción }\end{array}$ & $\begin{array}{c}\text { Bienes } \\
\text { de } \\
\text { Consumo }\end{array}$ & $\begin{array}{c}\text { Servicios } \\
\text { de } \\
\text { Consumo }\end{array}$ & $\begin{array}{c}\text { Servicios } \\
\text { Financieros e } \\
\text { Inmobiliarios }\end{array}$ & $\begin{array}{c}\text { Tecnología y } \\
\text { Telecomunicaciones }\end{array}$ & $\begin{array}{c}\text { TOTAL } \\
\text { EMPRESAS }\end{array}$ \\
\hline $\begin{array}{l}\text { Empresas con } \\
\text { código de } \\
\text { conducta visible }\end{array}$ & $\begin{array}{c}10 \\
(90,91 \%)\end{array}$ & $\begin{array}{c}23 \\
(69,70 \%)\end{array}$ & $\begin{array}{c}15 \\
(51,72 \%)\end{array}$ & $\begin{array}{c}11 \\
(61,11 \%)\end{array}$ & $\begin{array}{c}19 \\
(63,33 \%)\end{array}$ & $\begin{array}{c}7 \\
(87,50 \%)\end{array}$ & 85 \\
\hline $\begin{array}{l}\text { Empresas con } \\
\text { código de } \\
\text { conducta no } \\
\text { visible }\end{array}$ & 0 & 1 & 1 & 0 & 3 & 0 & 5 \\
\hline $\begin{array}{c}\text { Empresas sin } \\
\text { código de } \\
\text { conducta }\end{array}$ & 1 & 9 & 13 & 7 & 8 & 1 & 39 \\
\hline $\begin{array}{c}\text { TOTAL } \\
\text { EMPRESAS }\end{array}$ & 11 & 33 & 29 & 18 & 30 & 8 & 129 \\
\hline
\end{tabular}

Fuente: elaboración propia.

La tabla 1 permite observar como el sector de Petróleo y Energía y el sector de Tecnología y Telecomunicaciones son los sectores en los que las empresas cotizadas poseen en una amplia mayoría códigos éticos, pues en ambos del total de empresas cotizadas en el sector sólo una no posee código de conducta. En cambio, el sector de Bienes de Consumo, el sector de Materiales Básicos, Industria y Construcción junto al sector de Servicios Financieros e Inmobiliarios destacan por todo lo contrario, más el primero que los otros dos, pues cuentan con porcentajes altos de empresas sin código ético, y además son los únicos sectores con empresas que tienen códigos éticos no visibles. En cuanto al sector de Servicios de Consumo, ninguna empresa tiene código no visible pero es el segundo sector con menos empresas en posesión de un código ético.
En el estudio de Verona-Martel y Sosa-Breyer (2014: 31) también se concluye que en el sector de Bienes de Consumo se encuentra el mayor porcentaje de empresas cotizadas sin código ético, pues sólo poseen código de conducta el 29,03\% de las empresas. En cuanto al sector con mayor porcentaje de empresas con código de conducta, en el citado estudio se observa que es también el sector de Petróleo y Energía (90\%).

En relación a las empresas que entraron a cotizar en el año 2015 señalar que, de las ocho empresas que fueron incluidas en la Bolsa en el mercado continuo en dicho año, poseían código de conducta cinco de ellas (62,5\%), estando visibles dichos códigos. De estas cinco empresas, dos de ellas poseían código de conducta desde 2007 y 2008 respectivamente, en cambio las otras tres tienen código ético de fecha más reciente, concretamente desde 2015. 
De las empresas que no poseen código ético pertenecen dos de ellas al sector de Bienes de Consumo, sector que es el que cuenta con menos empresas que poseen código ético y la otra empresa pertenece al sector de Materiales Básicos, Industria y Construcción, sector que cuenta con un $69,70 \%$ de empresas que hayan publicado y hecho visible su código de conducta.

Señalar también que de estas tres empresas, las dos primeras a fecha de 2016 ya poseían código ético publicado y visible.

Centrándonos en el objetivo de este trabajo, a continuación se analiza en primer lugar el nivel de presencia en los códigos éticos de los tipos de acoso y discriminación que se rechazan en la empresa pero sin diferenciar por sectores económicos para, posteriormente, realizar el análisis por sectores de actividad.

De los 85 códigos éticos que forman la muestra objeto de estudio, en 81 (95,29\%) se hace referencia al rechazo al acoso y discriminación, presentándose detalle de qué tipo de acoso y discriminación la empresa no va a tolerar en 72 de ellos (84,71\%).

Sería aconsejable que las empresas especificaran de forma detallada qué tipo de acoso y discriminación no van a tolerar, y por supuesto más importante aún es que recojan que este tipo de comportamiento no está permitido en la empresa.
Verona-Martel y Sosa-Breyer (2014: 33) en su estudio sobre el contenido de los códigos éticos señalan que uno de los ítems que con mayor frecuencia se encuentran recogidos en ellos es el rechazo al acoso y discriminación, concretamente sus resultados muestran que aparece en un $96,67 \%$ de los códigos de conducta. Asimismo, estos autores señalan que los otros ítems recogidos en los códigos éticos con frecuencia superior al 90\% son: el rechazo a los conflictos de interés (96,67\%) y la prohibición de hacer pública información confidencial (93,33\%).

Los tipos de acoso y discriminación que las empresas recogen en su código ético indicando que no van a ser admitidos en la empresa se detallan en el cuadro 2.

Las empresas en el apartado de su código ético referido a la no tolerancia al acoso y a la discriminación indican que ello se refiere a la relación con y entre los empleados, señalando a su vez que buscan establecer un ambiente de trabajo libre de todo tipo de acoso y discriminación, especificando que no permiten que se cree un entorno de trabajo intimidatorio, ofensivo y hostil. Sólo de forma excepcional en algunos códigos éticos se añade a lo anterior que no se permitirá que los empleados acosen y/o discriminen a los grupos de interés (clientes y proveedores, por ejemplo).

Tal y como se observa en el cuadro 2 el rechazo a la discriminación por razón de sexo es el ítem más recogido en los códigos éticos, apareciendo en el $77,65 \%$ de los mismos. 
Cuadro 2. Tipos de acoso y discriminación recogidos en los códigos éticos

\begin{tabular}{|c|c|}
\hline Tipos de acoso y discriminación & $\begin{array}{c}\text { Presencia de los tipos de acoso y } \\
\text { discriminación en los códigos éticos (\%) }\end{array}$ \\
\hline Discriminación por razón de sexo & $77,65 \%$ \\
\hline Discriminación por raza/color & $68,24 \%$ \\
\hline Discriminación por religión & $67,06 \%$ \\
\hline Discriminación por nacionalidad & $61,18 \%$ \\
\hline Discriminación por edad & $48,24 \%$ \\
\hline Discriminación por orientación sexual & $44,71 \%$ \\
\hline Discriminación por discapacidad & $44,71 \%$ \\
\hline Acoso psicológico en el trabajo (mobbing) & $43,53 \%$ \\
\hline Acoso sexual & $42,35 \%$ \\
\hline Discriminación por ideología política & $40 \%$ \\
\hline Acoso físico & $35,29 \%$ \\
\hline Discriminación por origen o condición social & $29,41 \%$ \\
\hline Discriminación por estado civil & $25,88 \%$ \\
\hline Discriminación por afiliación sindical & $16,47 \%$ \\
\hline Discriminación por estado de salud & $8,23 \%$ \\
\hline Discriminación por idioma & $8,23 \%$ \\
\hline Discriminación por familiares a su cargo & $7,06 \%$ \\
\hline
\end{tabular}

Fuente: elaboración propia. 
Otros tipos de discriminación cuyo rechazo se recoge en más del $60 \%$ de los códigos éticos son: la discriminación por raza/color (68,24\%), por religión $(67,06 \%)$ y por nacionalidad (61,18\%). En cambio, los tipos de discriminación recogidos en estos códigos con menos frecuencia son la discriminación por estado de salud, por idioma y por tener familiares a su cargo; estos son recogidos en menos del $10 \%$ de los 85 códigos de conducta analizados en este trabajo.

Llama la atención que el ítem más destacado sea la discriminación por razón de sexo cuando por ejemplo en la alta dirección y en el consejo de administración de las empresas la participación femenina en ambos órganos es muy baja. Tal es así que en determinadas normativas se recoge la recomendación de que se incorporen más mujeres al consejo de administración ${ }^{1}$, y entre estas se pueden citar la Ley Orgánica 3/2007, de 22 de marzo, para la igualdad efectiva de mujeres y hombres (ver artículos 54, 75 y 78) y el Código de Buen Gobierno español de 2015 (ver Principio 10 y Recomendación 14). Estas normativas son en territorio español y no obligan sino recomiendan esa mayor participación de la mujer en el consejo de administración de las empresas.

En España a fecha de último día de diciembre de 2015 el peso medio de la mujer en el consejo de administración de las empresas cotizadas en Bolsa en el mercado continuo se situaba en un $15,30 \%$, y poseían mujeres consejeras un $80,62 \%$ de las empresas cotizadas. La situación con respecto a la participación femenina en el equipo de alta dirección ofrece a tal fecha un panorama más negativo, pues el peso medio de la mujer era de un $11,47 \%$, y tenían mujeres sentadas en su equipo de alta dirección un 59,69\% de las empresas cotizadas.

A lo anterior es preciso añadir que la Ley Orgánica 3/2007 recomendaba que para el año 2015 en el consejo de administración las personas de cada sexo no superasen el $60 \%$ ni fuesen menos del $40 \%$. Sin embargo, en ese año sólo cumplían tal requisito tres empresas.

El acoso sexual y el acoso psicológico en el trabajo (mobbing) han sido puestos de manifiesto en la literatura que afectan más a las mujeres que a los hombres (Pernas y Ligero, 2003: 9-11; Moreno Jiménez et al., 2005: 8; Escartín et al., 2008: 208; González Trijueque y Graña Gómez, 2009: 292; Carnero et al., 2010: 3784; Pérez Guardo y Rodríguez Sumaza, 2013: 215), y ambos están recogidos en el Código Penal español como delitos (ver artículos 173 y 184), no obstante sólo aparecen recogidos en menos del $50 \%$ de los códigos éticos, concretamente el mobbing en un 43,53\% y el acoso sexual en un $42,35 \%$ de los códigos éticos de las empresas cotizadas.

En una sociedad democrática son derechos básicos la libertad de ideología política y la afiliación sindical, en cambio la primera sólo aparece recogida en el $40 \%$ de los códigos éticos y la segunda ni en el $17 \%$ de dichos códigos, concretamente en el 16,47\%.

El Estatuto de los Trabajadores recoge los derechos laborales de todo trabajador y se observa que de tales derechos aparecen más de la mitad de ellos recogidos en los códigos éticos en un porcentaje igual o superior a un 40\% (derecho a no ser discriminado por razón de sexo, por raza, por religión, por edad, por orientación sexual, por discapacidad, por ideología política y a no sufrir acoso sexual). Los que aparecen con un porcentaje inferior al $40 \%$ son los derechos a no ser discriminado por condición social, por afiliación sindical, por idioma ni a sufrir acoso físico. Sin embargo, en este Estatuto no se recoge la no discriminación por nacionalidad y en cambio es un ítem recogido en el 61,18\% de los códigos éticos. Tampoco recoge el Estatuto de los Trabajadores

\footnotetext{
${ }^{1}$ Las normativas existentes en España se centran en recomendar incrementar la presencia femenina en el consejo de administración pero no hacen referencia a la alta dirección. A este respecto Gabaldón (2013: 46) afirma que si bien es “relevante ver cómo la presión legislativa ha hecho cambiar los consejos de administración no parece que esté siendo totalmente permeable hacia abajo, dado que las primeras líneas de dirección son aún mayoritariamente masculinas”.
} 
una referencia explícita al acoso psicológico en el trabajo (mobbing), y está recogido en el $43,53 \%$ de los códigos éticos.

En cuando a los derechos recogidos en la Constitución Española, todos están recogidos en los códigos éticos pero no con porcentajes similares, pues aparecen en un porcentaje del $40 \%$ o superior el derecho a no ser discriminado por sexo, por raza, por religión, por ideología política y a no sufrir mobbing, mientras que los derechos a la libertad de sindicación y el no sufrir acoso físico están recogidos en menos del $40 \%$ de los códigos éticos. La Constitución sí hace una referencia explícita al acoso psicológico en el trabajo pero deja fuera otros tipos de discriminación que se pueden sufrir y que sí aparecen recogidos en un alto porcentaje en los códigos éticos como el acoso sexual y la discriminación por edad, por orientación sexual y por discapacidad.

El cuadro 3 recoge la información referida a los tipos de acoso y discriminación por sectores económicos.

El sector de Petróleo y Energía tiene de las 11 empresas que engloba 10 con código ético visible, teniendo el $70 \%$ de los códigos fecha de publicación anterior a 2008, habiendo sufrido al menos una revisión antes del 2014 y datando de 2002 el primer código ético publicado.

En cuanto a su contenido, el 100\% de los códigos éticos en este sector consideran el tema del rechazo al acoso y a la discriminación, pero sólo el $80 \%$ presenta detalle al respecto, es decir, especifica qué tipos de acoso y discriminación no son tolerados en la empresa.

Todos los tipos de acoso y discriminación analizados en este trabajo están presentes en los códigos éticos de este sector con un mayor peso que en la muestra general a excepción de cinco de ellos: discriminación por nacionalidad, por discapacidad, por afiliación sindical, por estado civil y por familiares a su cargo. En relación a la discriminación por discapacidad se da el hecho de que este es el sector que menos recoge en sus códigos éticos el rechazo a este tipo de discriminación, y en cuanto a la discriminación por afiliación sindical es el segundo. Asimismo, no se recogen en los códigos éticos de este sector el rechazo a la discriminación por familiares a su cargo.

Hay que destacar la importancia en relación a la muestra total que se da en los códigos éticos de este sector al rechazo a la discriminación por edad, al mobbing, a la discriminación por orientación sexual y a la discriminación por ideología política, todos con porcentajes iguales o superiores al $60 \%$ cuando en la muestra total tienen porcentajes inferiores al 50\%. Asimismo, este sector es el que más importancia da en sus códigos éticos al rechazo a la discriminación por ideología política y por estado de salud. También, es de señalar la importancia otorgada al rechazo por discriminación por raza/color, por religión, por origen y por idioma. 
Cuadro 3. Tipos de acoso y discriminación recogidos en los códigos éticos por sectores de actividad económica

\begin{tabular}{|c|c|c|c|c|c|c|c|}
\hline Tipos de acoso y discriminación & $\begin{array}{c}\text { Petróleo } \\
\text { y Energía } \\
(\%)\end{array}$ & $\begin{array}{c}\text { Mat. } \\
\text { Básicos, } \\
\text { Industria } \\
\text { y Cons- } \\
\text { trucción } \\
\text { (\%) }\end{array}$ & $\begin{array}{c}\text { Bienes } \\
\text { de } \\
\text { Consumo } \\
\text { (\%) }\end{array}$ & $\begin{array}{c}\text { Servicios } \\
\text { de } \\
\text { Consumo } \\
\text { (\%) }\end{array}$ & $\begin{array}{c}\text { Servicios } \\
\text { Finan- } \\
\text { cieros e } \\
\text { Inmo- } \\
\text { biliarios } \\
\text { (\%) }\end{array}$ & $\begin{array}{c}\text { Tecno- } \\
\text { logía y } \\
\text { Teleco- } \\
\text { munica- } \\
\text { ciones } \\
(\%)\end{array}$ & $\begin{array}{c}\text { MUESTRA } \\
\text { TOTAL } \\
(\%)\end{array}$ \\
\hline Discriminación por razón de sexo & $80 \%$ & $69,57 \%$ & $80 \%$ & $81,82 \%$ & $73,68 \%$ & $100 \%$ & $77,65 \%$ \\
\hline Discriminación por raza/color & $80 \%$ & $60,87 \%$ & $66,67 \%$ & $72,73 \%$ & $63,16 \%$ & $85,71 \%$ & $68,24 \%$ \\
\hline Discriminación por religión & $80 \%$ & $60,87 \%$ & $53,33 \%$ & $81,82 \%$ & $57,89 \%$ & $100 \%$ & $67,06 \%$ \\
\hline Discriminación por nacionalidad & $60 \%$ & $52,17 \%$ & $66,67 \%$ & $63,64 \%$ & $57,89 \%$ & $85,71 \%$ & $61,18 \%$ \\
\hline Discriminación por edad & $70 \%$ & $39,13 \%$ & $46,67 \%$ & $64,64 \%$ & $31,58 \%$ & $71,43 \%$ & $48,24 \%$ \\
\hline $\begin{array}{l}\text { Acoso psicológico en el trabajo } \\
\text { (mobbing) }\end{array}$ & $70 \%$ & $43,48 \%$ & $40 \%$ & $72,73 \%$ & $15,79 \%$ & $42,86 \%$ & $43,53 \%$ \\
\hline $\begin{array}{l}\text { Discriminación por orientación } \\
\text { sexual }\end{array}$ & $60 \%$ & $26,09 \%$ & $26,67 \%$ & $81,82 \%$ & $36,84 \%$ & $85,71 \%$ & $44,71 \%$ \\
\hline Discriminación por discapacidad & $30 \%$ & $34,78 \%$ & $40 \%$ & $63,64 \%$ & $42,11 \%$ & $85,71 \%$ & $44,71 \%$ \\
\hline Acoso sexual & $50 \%$ & $43,48 \%$ & $40 \%$ & $45,45 \%$ & $42,11 \%$ & $28,57 \%$ & $42,35 \%$ \\
\hline $\begin{array}{l}\text { Discriminación por ideología } \\
\text { política }\end{array}$ & $60 \%$ & $39,13 \%$ & $20 \%$ & $54,55 \%$ & $31,58 \%$ & $57,14 \%$ & $40 \%$ \\
\hline Acoso físico & $40 \%$ & $34,78 \%$ & $33,33 \%$ & $54,55 \%$ & $31,58 \%$ & $14,29 \%$ & $35,29 \%$ \\
\hline $\begin{array}{l}\text { Discriminación por origen o } \\
\text { condición social }\end{array}$ & $40 \%$ & $34,78 \%$ & $6,67 \%$ & $36,36 \%$ & $26,32 \%$ & $42,86 \%$ & $29,41 \%$ \\
\hline
\end{tabular}


Cuadro 3. Continuación...

\begin{tabular}{|l|l|l|l|l|l|l|l|}
\hline & \multicolumn{1}{|c|}{$\begin{array}{c}\text { Petróleo } \\
\text { Tipos de acoso y discriminación } \\
\mathbf{y} \text { Energía } \\
\mathbf{( \% )}\end{array}$} & $\begin{array}{c}\text { Básicos, } \\
\text { Industria } \\
\mathbf{y} \text { Cons- } \\
\text { trucción } \\
\mathbf{( \% )}\end{array}$ & $\begin{array}{c}\text { Bienes } \\
\mathbf{d e} \\
\text { Consumo } \\
\mathbf{( \% )}\end{array}$ & $\begin{array}{c}\text { Servicios } \\
\mathbf{d e} \\
\text { Consumo } \\
\mathbf{( \% )}\end{array}$ & $\begin{array}{c}\text { Servicios } \\
\text { Finan- } \\
\text { cieros e } \\
\text { Inmo- } \\
\text { biliarios } \\
\mathbf{( \% )}\end{array}$ & $\begin{array}{c}\text { Tecno- } \\
\text { logía y } \\
\text { Teleco- } \\
\text { munica- } \\
\text { ciones }\end{array}$ & $\begin{array}{c}\text { MUESTRA } \\
\text { TOTAL } \\
\mathbf{( \% )}\end{array}$ \\
\hline Discriminación por estado civil & $20 \%$ & $13,04 \%$ & $13,33 \%$ & $54,54 \%$ & $26,32 \%$ & $57,14 \%$ & $25,88 \%$ \\
\hline $\begin{array}{l}\text { Discriminación por afiliación } \\
\text { sindical }\end{array}$ & $10 \%$ & $8,70 \%$ & $26,67 \%$ & $18,18 \%$ & $15,79 \%$ & $28,57 \%$ & $16,7 \%$ \\
\hline $\begin{array}{l}\text { Discriminación por estado de } \\
\text { salud }\end{array}$ & $30 \%$ & $4,35 \%$ & $0 \%$ & $9,09 \%$ & $10,52 \%$ & $0 \%$ & $8,23 \%$ \\
\hline Discriminación por idioma & $20 \%$ & $4,35 \%$ & $0 \%$ & $9,09 \%$ & $5,26 \%$ & $28,57 \%$ & $8,23 \%$ \\
\hline $\begin{array}{l}\text { Discriminación por familiares a su } \\
\text { cargo }\end{array}$ & $0 \%$ & $0 \%$ & $6,67 \%$ & $18,18 \%$ & $0 \%$ & $42,86 \%$ & $7,06 \%$ \\
\hline
\end{tabular}

Fuente: elaboración propia.

El sector de Materiales Básicos, Industria y Construcción cuenta con 33 empresas cotizadas de las cuales tienen código ético visible 23 empresas y una posee un código ético pero no es visible. El primer código ético tiene fecha de publicación de 2003, el 43,48\% de los códigos éticos visibles fueron publicados antes de 2010 (26,09\% antes del 2008), y han sufrido alguna revisión un 39,13\% de los códigos de conducta visibles de este sector.

En relación a su contenido, el 86,96\% hacen referencia al rechazo al acoso y discriminación, presentando esta información detallada el 78,26\%.
En el análisis de los tipos de acoso y discriminación recogidos en los códigos éticos de este sector se observa que, excepto para la discriminación por origen o condición social y el acoso sexual, los porcentajes son inferiores a los de la muestra total aunque los tipos de discriminación que tienen una mayor relevancia en la muestra total (discriminación por razón de sexo, por raza/color, por religión y por nacionalidad), también la tienen en este sector. 
Es destacar que los tipos de discriminación que en este sector tienen unos valores considerablemente menores que en la muestra total son la discriminación por orientación sexual y por estado civil. De hecho es este sector en el que el rechazo a la discriminación por orientación sexual y por estado civil está menos presente en los códigos éticos. También destaca este sector por ser en el que menos se recoge en los códigos éticos el rechazo a la discriminación por razón de sexo, por raza/color, por nacionalidad y por afiliación sindical. Asimismo, no se recogen en los códigos éticos de este sector el rechazo a la discriminación por familiares a su cargo.

El sector de Bienes de Consumo con 29 empresas cotizadas sólo cuenta con 15 empresas con código visible y con una empresa con código de conducta no visible. El primer código de conducta fue publicado en 2001, el 33,33\% de los códigos éticos visibles fueron publicados antes del 2010 (26,67\% antes del 2008), y han sufrido alguna revisión un 33,33\% del total de códigos éticos visibles en este sector.

En relación a su contenido, el 100\% de los códigos éticos hace referencia al rechazo al acoso $\mathrm{y}$ discriminación pero sólo presentan el $80 \%$ de ellos detalle al respecto.

En cuanto a los porcentajes que presentan los distintos tipos de acoso y discriminación estudiados, en este sector en relación a la muestra total se observa que en todos excepto en tres (el rechazo a la discriminación por razón de sexo, por nacionalidad y por afiliación sindical), presenta valores inferiores.

En este sector se obtienen los valores más bajos para cincos tipos de acoso y discriminación, a saber: discriminación por religión, por ideología política, por origen o condición social, por idioma y por mobbing. También destacan al compararse con la muestra total los bajos valores que toma la discriminación por orientación sexual y por estado civil.
Asimismo, en este sector hay dos tipos de discriminación cuyo rechazo no se recoge en los códigos éticos: la discriminación por estado de salud y por idioma.

El sector de Servicios de Consumo cuenta con 11 empresas que tienen código visible de las 18 que cotizan en el mismo. El código de conducta con fecha de publicación más antigua data de 2008 y antes del 2010 sólo tres empresas tenían publicado código ético; es un sector en el que los códigos éticos comienzan a publicarse a partir del 2010. Tan sólo dos empresas, las que publicaron su código ético en 2008, han hecho revisiones del mismo.

En cuanto al contenido de estos códigos éticos se puede decir que el $100 \%$ de ellos recoge el rechazo al acoso y discriminación y presenta detalle al respecto.

Si se comparan los porcentajes de los distintos tipos de acoso y discriminación recogidos en estos códigos éticos con la muestra general se observa que para todos los ítems considerados los valores son más altos.

Este sector es el que da más importancia al rechazo al acoso psicológico en el trabajo (la denominada lacra laboral del siglo XXI), al acoso físico y a la discriminación por orientación sexual. También, destaca la importancia otorgada en los códigos éticos de este sector al rechazo a la discriminación por edad, por discapacidad, por ideología política, por estado civil y por familiares a su cargo.

En el sector de Servicios Financieros e Inmobiliarios sólo 19 empresas tienen código visible de las 30 cotizadas, siendo el sector con más empresas que tienen código no visible, un total de tres empresas. El primer código ético publicado data del año 2000 y antes del 2010 el 42,11\% de las empresas tenían código ético publicado visible (31,58\% antes del año 2008). Asimismo, el 26,32\% de las empresas con código ético visible han realizado alguna revisión en el mismo. 
En relación al contenido, el 94,74\% de los códigos éticos en este sector recogen el rechazo al acoso y discriminación, presentando detalle un 84,21\%.

Este sector presenta unos valores inferiores para todos los tipos de acoso y discriminación analizados en comparación con la muestra total, excepto en el rechazo a la discriminación por estado civil y por estado de salud.

Destaca la poca importancia que se otorga en los códigos de este sector al rechazo al mobbing con un porcentaje del $15,79 \%$ cuando en la muestra general toma un valor del $43,53 \%$, y en el resto de sectores se sitúa el acoso psicológico en el trabajo con unos valores iguales o superiores al $40 \%$.

También se da una importancia mucho más baja que en la muestra general al rechazo a la discriminación por religión, por edad, por orientación sexual y por ideología política. Asimismo, no se recoge en ninguno de los códigos de este sector el rechazo a la discriminación por familiares a su cargo; este es el único ítem que no aparece recogido en ninguno de los códigos de conducta de tres sectores económicos.

El sector de Tecnología y Telecomunicaciones tiene 7 empresas de las 8 cotizadas con código ético visible, siendo la fecha de publicación del primer código ético del año 2000, código que es el único que ha sufrido revisiones del total de códigos éticos de este sector. Los códigos éticos de las empresas de este sector fueron publicados fundamentalmente después del año 2009 pues antes del 2008 sólo dos empresas tenían código ético, uniéndose a este grupo otra empresa en 2009, mientras que el resto tienen sus códigos éticos publicados en 2011, 2014 y 2015.

En cuanto al contenido de los códigos éticos de este sector, el 100\% tienen recogidos el rechazo al acoso y discriminación presentando todos detalle al respecto.

Los valores que presentan los distintos tipos de acoso y discriminación en este sector son mayores que los recogidos para la muestra total, excepto para el acoso psicológico en el trabajo, el acoso sexual, el acoso físico y la discriminación por estado de salud. Tanto para el acoso sexual como para el acoso físico este sector presenta los porcentajes más bajos de todos los sectores, y en relación a la discriminación por estado de salud simplemente no es considerada en ninguno de los códigos éticos.

Este sector es el que otorga en sus códigos éticos mayor importancia al rechazo por discriminación por razón de sexo y por religión (ambos incluidos en todos sus códigos éticos), por raza/color, por nacionalidad, por edad, por orientación sexual, por discapacidad, por origen o condición social, por estado civil, por afiliación sindical, por idioma y por familiares a su cargo.

Por tanto, de los 17 tipos de acoso y discriminación considerados en el estudio este sector tiene los mayores porcentajes de todos los sectores para 12 de ellos, sólo tiene porcentajes inferiores a los de la muestra total para 4, y para el restante ítem (que es el rechazo a la discriminación por ideología política) su porcentaje es superior al obtenido en la muestra total. 


\section{Conclusiones}

El objetivo del presente estudio es analizar los tipos de acoso y discriminación recogidos en los códigos de conducta o códigos éticos de las empresas cotizadas en la Bolsa española en el mercado continuo a 31 de diciembre de 2015. Los resultados obtenidos en este trabajo permiten extraer las siguientes conclusiones:

- Los códigos de conducta van ganando importancia entre las empresas con el paso del tiempo. De las 129 empresas cotizadas en la Bolsa española a último día del 2015 cuentan con código ético visible 85 de ellas, las cuales los recogen en su página web corporativa, habiendo sido muchos de ellos publicados o bien revisados en los últimos cinco años.

- El ítem referido a la no tolerancia a la discriminación y el acoso aparece recogido en la mayoría de los códigos analizados (95,29\%), presentando el $84,71 \%$ de estos códigos información detallada al respecto.

- Los tipos de acoso y discriminación más recogidos en los códigos éticos que las empresas declaran no permitir son: la discriminación por razón de sexo (77,65\%), la discriminación por raza/color $(68,24 \%)$ y la discriminación por religión (67,06\%). Los menos frecuentes son: la discriminación por estado de salud (8,23\%), por idioma $(8,23 \%)$ y por familiares a su cargo (7,06\%).

- Las empresas englobadas en el sector de Bienes de Consumo son las que presentan peor índice de posesión de código de conducta, pues de 29 empresas cotizadas pertenecientes a este sector sólo 15 empresas poseen código ético visible (51,72\%). En cambio, los sectores de Petróleo y Energía y el de Tecnología y Telecomunicaciones destacan por todo lo contrario, ya que en ambos del total de empresas cotizadas en el sector sólo una no cuenta con código ético, por lo que sus porcentajes de empresas con código de conducta son elevados, un $90,91 \%$ y un $87,50 \%$, respectivamente.

- El análisis por sectores de actividad de los tipos de acoso y discriminación recogidos en los códigos éticos pone de manifiesto que en algunos sectores se da más importancia al rechazo a unos tipos y menos a otros, incluso se observa como determinados ítems no son recogidos en algunos sectores ni en un solo código ético. Si bien, en todos los sectores se observa que el rechazo a la discriminación por razón de sexo es el más relevante, aunque en algunos sectores comparte este puesto con otros tipos de discriminación como la discriminación por religión, por raza/color o por orientación sexual.

La ética en las empresas es un tema que está ganando creciente importancia, ya que la sociedad actual se cuestiona cada vez más los valores y principios morales en el ámbito de los negocios. En este aspecto, se ha avanzado en la transparencia de las empresas por mostrar sus valores éticos, dato reflejado en este trabajo a través del número de empresas que poseen código ético y la amplitud de los tipos de acoso y discriminación recogidos en este documento que la empresa declara rechazar, pero a pesar de ello quedan muchos objetivos por conseguir.

Tras este estudio se podrían llevar a cabo otras líneas de investigación relacionadas, como el grado de acoso y discriminación que puede existir en la empresa y la eficacia que tienen los códigos de conducta para impedir que se realicen los tipos de prácticas y comportamientos analizados en este trabajo.

\section{Referencias}

Carnero, M. A.; Martínez, B.; Sánchez-Mangas, R. (2010). Mobbing and its determinants: the case of Spain. Applied Economics, 42 (29), pp. 3.777-3.787. 
Comisión Nacional del Mercado de Valores (2015). Código de buen gobierno de las sociedades cotizadas. Comisión Nacional del Mercado de Valores. España.

Constitución Española (1978). BOE núm. 311 de 29 de diciembre de 1978.

Cortina, A. (1998). Hasta un pueblo de demonios. Ética pública y sociedad. Madrid: Ed. Taurus.

Cortina, A. (coord.) (2003). Ética de la empresa. Claves para una nueva cultura empresarial. $6^{\circ}$ edición. Madrid: Editorial Trotta.

Debeljuh, P. (2008). Los códigos de ética en las empresas. Revista Economia \& Gestão, 5 (9), pp. 4856.

Escartín, J.; Rodríguez-Caballeira, A.; Porrúa, C.; Martín-Peña, J. (2008). Estudio y análisis sobre cómo perciben el mobbing los trabajadores. Revista de Psicología Social, 23 (2), pp. 203-211.

Gabaldón, P. (2013). Mujeres en los Consejos de Administración y en la Alta Dirección en España. Investigaciones y publicaciones 2013 (I). Centro de Gobierno Corporativo. IE Business School. España.

García Sánchez, I. M.; Rodríguez Domínguez, L.; Gallego Álvarez, I. (2008). La influencia del Consejo de Administración en la adopción de un código ético, Revista de Contabilidad-Spanish Accounting Review, 11 (2), pp. 93-120.

González Trijueque, D.; Graña Gómez, J. L. (2009). El acoso psicológico en el lugar de trabajo: prevalencia y análisis descriptivo en una muestra multiocupacional. Psicothema, 21 (2), pp. 288-293.

Iranzo, J. E. (2004). El fortalecimiento de la cultura de la ética empresarial como mecanismo de autocontrol. Economistas, pp. 268-271.

Ley Orgánica 10/1995, de 23 de noviembre, del Código Penal. Última modificación: 2 de marzo de 2019. España.
Ley Orgánica 3/2007, de 22 de marzo, para la igualdad efectiva de mujeres y hombres. España.

Lozano Aguilar, J. F. (2004). Códigos éticos para el mundo empresarial. Madrid: Ed. Trotta.

Lozano Aguilar, J. F. (2007). Códigos éticos y auditorías éticas. Veritas, 2 (17), pp. 225-251.

Moreno Jiménez, B.; Rodríguez Muñoz, A.; Garrosa Hernández, E.; Morante Benadero, M. (2005). Antecedentes organizacionales del acoso psicológico en el trabajo: un estudio exploratorio. Psicothema, 17 (4), pp. 627-632.

Pérez Guardo, R.; Rodríguez Sumaza, C. (2013). Un análisis del concepto de acoso sexual laboral: reflexiones y orientaciones para la investigación y la intervención social. Cuadernos de Relaciones Laborales, 31 (1), pp. 195-219.

Pernas, B.; Ligero, J. A. (2003). Más allá de una anomalía: el acoso sexual en la encrucijada entre sexualidad y trabajo. En Raquel Osborne y Oscar Guasch (comps.): Sociología de la sexualidad. Madrid: CIS-Siglo XXI, Colección Monografías, $\mathrm{n}^{0}$ 195, pp. 126-158.

Real Decreto Legislativo 2/2015, de 23 de octubre, por el que se aprueba el texto refundido de la Ley del Estatuto de los Trabajadores. España.

Regojo, P. (2014). Ética para directivos y consejeros. Cómo construir empresas excelentes y socialmente responsables. Navarra: Ediciones Universidad de Navarra, S.A. (EUNSA).

Valor, C.; De la Cuesta González, M. (2007). Códigos éticos: análisis de la eficacia de su implantación entre las empresas españolas cotizadas. Innovar, 17 (30), pp. 19-30.

Verona-Martel, M. C.; Sosa-Breyer, M. (2014). Códigos éticos de las empresas cotizadas españolas: análisis de su contenido. Vértice Universitario, (61), pp. 23-38. 